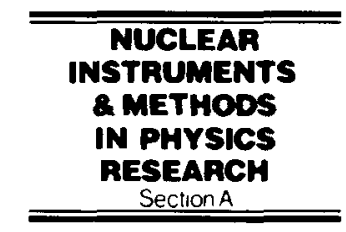

EISEVIER

\title{
Quasiparticle lifetimes and tunneltimes in SIS junctions for X-ray spectroscopy
}

\author{
O.J. Luiten ${ }^{a, *}$, H.L. van Lieshout ${ }^{a}$, F.A. Michels ${ }^{a}$, P. Valko ${ }^{\text {a.l }}$, M.P. Bruijn ${ }^{a}$, F. Kiewiet ${ }^{a}$, \\ P.A.J. de Korte ${ }^{\mathrm{a}}$, D.J. Adelerhof ${ }^{\mathrm{b}}$, A.W. Hamster ${ }^{\mathrm{b}}$, C.G.S. Brons ${ }^{\mathrm{b}}$, J. Flokstra ${ }^{\mathrm{b}}$

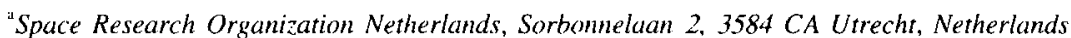

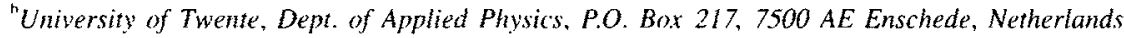

\begin{abstract}
The quasiparticle lifetimes and tunneltimes in SIS tunnel junctions are essential parameters in the development of these devices for high resolution X-ray spectroscopy. We present a simple analytical model which allows us to calculate both the risetime and the total charge of the integrated tunnel current following an $X$-ray event. Simultaneous measurement of both the total charge and the risetime thus becomes a useful diagnostic tool for quantitative analysis. We have applied the model to recent X-ray measurements on a Nb/AlO, SIS junction. We find that the energy resolution achieved in this device is mainly limited by variations in the thickness of the counter electrode.
\end{abstract}

\section{Introduction}

Intrinsic variation of the quasiparticle lifetimes and tunneltimes in SIS tunnel junction $\mathrm{X}$-ray detectors is generally considered to be the cause for the failure sofar to reach the goal of $10 \mathrm{eV}$ FWHM energy resolution for $6 \mathrm{keV}$ photons [1].

In this article we present a linear 1-dimensional model which takes the finite lifetimes and tunneltimes in both electrodes of an SIS junction into account, therefore including back tunneling. Analytical expressions are derived, which enable a relatively straightforward calculation of the effect of variation of the different time constants on the observed total charge and risetime. Non-linear behavior and finite lateral size effects, due to the recombination and the diffusion of the quasi-particles, are neglected. This simplified model has the advantage of only a few parameters and the clarity and convenience of an analytical approach.

\section{Theory}

We describe the evolution of the quasiparticle populations $N_{1}$ and $N_{2}$ in, respectively, electrode 1 and electrode 2 of an SIS tunnel junction by

\footnotetext{
* Corresponding author. Tel. +31 30 535637, fax +31 30 540860 , e-mail j.luiten@sron.ruu.nl.

'Present address: Slovak Techn. University, Dept. of Physics, Ilkovicava 3, 812 19 Bratislava, Slovak Republic.
}

$\dot{N}_{1}=-\left(\tau_{1,1}^{-1}+\tau_{1.1}^{-1}\right) N_{1}+\tau_{t, 2}^{-1} N_{2}$,
$\dot{N}_{2}=-\left(\tau_{1,2}^{-1}+\tau_{1.2}^{-1}\right) N_{2}+\tau_{1,1}^{-1} N_{1}$,

where $\tau_{t, 1(2)}$ is the tunneltime from electrode 1(2) to electrode $2(1)$ and $\tau_{1,1,2,}$ is the quasi-particle lifetime in electrode 1(2). The corresponding tunnel current $I_{\mathrm{t}}$ is then given by

$\frac{I_{\mathrm{t}}(t)}{e}=\frac{N_{1}(t)}{\tau_{\mathrm{t}, 1}}+\frac{N_{2}(t)}{\tau_{\mathrm{t}, 2}}$.

The experimentally observed quantity is the collected charge as a function of integration time:

$Q(t)=\int_{0}^{t} \mathrm{~d} t^{\prime} I_{\mathrm{r}}\left(t^{\prime}\right)$

Given the initial condition $N_{1}(0)=N_{0}$ and $N_{2}(0)=0$, for instance due to the absorption of a $6 \mathrm{keV} \mathrm{X}$-ray photon $\left(N_{0}=2.4 \times 10^{6}[2,3]\right)$ in electrode 1 , the set of equations (1) is solved analytically. The result is:

$$
\begin{aligned}
\frac{Q(t)}{Q_{0}}= & A\left[1-\left(\cosh y t+\frac{x}{y} \sinh y t\right) \mathrm{e}^{-x t}\right] \\
& +B\left[1-\left(\cosh y t+\frac{y}{x} \sinh y t\right) \mathrm{e}^{-x t}\right],
\end{aligned}
$$

where

$Q_{0}=N_{0} e$,

$A=\frac{c_{1}\left(3 c_{2}-c_{1}-c_{3}+c_{4}\right)}{2\left(c_{1} c_{4}+c_{2} c_{3}+c_{3} c_{4}\right)}$,

$B=\frac{c_{1}\left(c_{1}+c_{2}+c_{3}+c_{4}\right)}{2\left(c_{1} c_{4}+c_{2} c_{3}+c_{3} c_{4}\right)}$. 
$x=\left(c_{1}+c_{2}+c_{3}+c_{4}\right) / 2$,

$y=\left[\left(c_{1}^{2}+c_{2}^{2}+c_{3}^{2}+c_{4}^{2}+2 c_{1} c_{2}+2 c_{1} c_{3}-2 c_{2} c_{3}\right.\right.$

$$
\left.\left.-2 c_{1} c_{4}+2 c_{2} c_{4}-2 c_{3} c_{4}\right)^{1 / 2}\right] / 2 \text {, }
$$

with $c_{1,2)}=\tau_{t, 1(2)}^{-1}$ and $c_{3(4)}=\tau_{1,1(2)}^{-1}$. From Eq. (4) the tunneled charge per event is obtained:

$\frac{Q(\infty)}{Q_{0}}=\frac{2 c_{1} c_{2}+c_{1} c_{4}}{c_{1} c_{4}+c_{2} c_{3}+c_{3} c_{4}}$.

which corresponds to the expression derived by Goldie et al. [4].

It is instructive to consider two limiting cases: (i) the case of a nearly completely "killed" counter electrode, i.e. $\tau_{1,2} \ll \tau_{1,1}, \tau_{t, 1}, \tau_{t, 2}$, and (ii) the case of a completely symmetrical junction, i.e. $\tau_{\mathrm{t}, 1}=\tau_{\mathrm{t}, 2}=\tau_{\mathrm{r}}$ and $\tau_{1,1}=\tau_{1,2}=\tau_{1}$.

(i) For small values of $\tau_{1,2}$, Eq. (4) may be expanded to first order in $\tau_{1,2} / \tau_{1,1}, \tau_{1,2} / \tau_{1,1}$, and $\tau_{1,2} / \tau_{\mathrm{t}, 2}$, yielding:

$\frac{Q_{1}(t)}{Q_{0}}=\frac{\tau_{\mathrm{r}}}{\tau_{\mathrm{t} .1}}\left(1+\frac{\tau_{1.2}}{\tau_{\mathrm{t} .2}}\right)\left(1-\mathrm{e}^{-t / \tau_{\mathrm{r}}}\right)$,

with the risetime $\tau_{\mathrm{r}}$ given by

$\tau_{\mathrm{r}}^{-1}=\tau_{\mathrm{t}, 1}^{-1}\left(1-\frac{\tau_{1,2}}{\tau_{1,2}}\right)+\tau_{1,1}^{-1}$

For exact "one way traffic" of the quasiparticles $\left(\tau_{1,2}=0\right)$, Eqs. (7) and (8) reduce to familiar expressions.

(ii) For symmetric "two way traffic" of the quasiparticles Eq. (4) simply reduces to

$\frac{Q_{2}(t)}{Q_{0}}=\frac{\tau_{1}}{\tau_{\mathrm{t}}}\left(1-\mathrm{e}^{-t / \tau_{1}}\right)$,

so in this case the risetime is equal to the lifetime $\tau_{1}$.

As is clear from Eq. (4), the evolution of $Q(t)$ in general is governed by the two time constants $(x-y)^{-1}$ and $(x+y)^{-1}$, in contrast with the two special cases (i) and (ii) treated above. A proper general definition of the risetime is therefore not immediately obvious. For comparison with experimental data we use an operational definition of the risetime: $\tau_{\mathrm{r}}=\left(\begin{array}{ll}\tau_{0 . k} & \tau_{0.4}\end{array}\right) / 1.1$, with $Q\left(\tau_{\alpha}\right)=\alpha Q(\infty)$ (for $\alpha=0.8$ and 0.4 ), which is equivalent to the way we determine the risetime experimentally.

\section{Junction response dependence of the various time constants}

In order to investigate the influence of variation of the lifetimes and the tunneltimes on the junction response, we have varied each of the four tinte constants separately. The result is plotted in Fig. 1 in the form of four risetimecharge diagrams. The charge $Q(\infty)$ is calculated using Eq. (6) and the risetime $\tau_{\mathrm{r}}$ using Eq. (4) and the recipe given at the end of the previous section. For each set of parameters

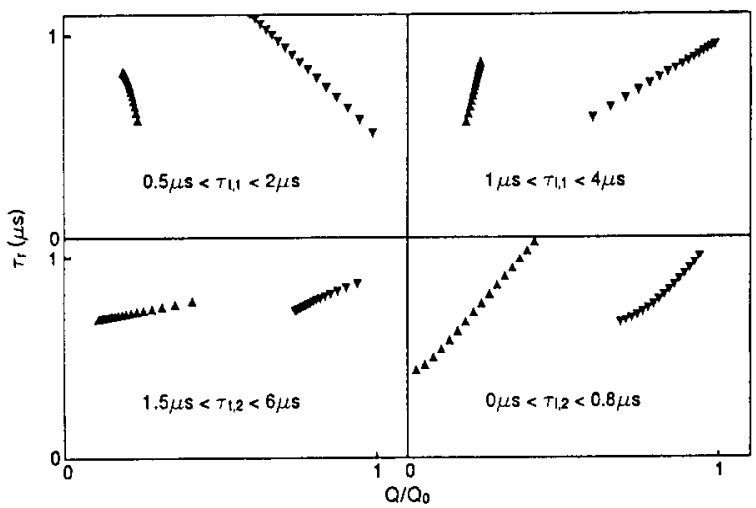

Fig. 1. Risetime-charge diagrams showing the effect of the separate variation of each of the four time constants on the base (down triangles) and the counter electrode (up triangles) signal. The triangles are separated by equal amounts of the variable parameter.

the response is calculated for $\mathrm{X}$-ray events both in the base and the counter electrode.

The calculation is centered on the parameter set $\tau_{1,1}=$ $2 \mu \mathrm{s}, \tau_{\mathrm{t} .1}=1 \mu \mathrm{s}, \tau_{1.2}=0.4 \mu \mathrm{s}$, and $\tau_{\mathrm{t}, 2}=3 \mu \mathrm{s}$, which is a reasonable set of values for the junction used in the experiments (see Section 4). In each diagram one of the four timc constants is varied around the given value. Fig. 1 clearly shows that the influence of each of the separate time constants is different and sometimes quite non-trivial: For example, variation of $\tau_{t, 1}$ results in trajectories in phase space roughly perpendicular to those due to variation of $\tau_{t, 2}$.

\section{Comparison with experiment}

Recently, we have performed X-ray measurements on a $140 \mu \mathrm{m} \times 140 \mu \mathrm{m} \mathrm{Nb} / \mathrm{AlO}_{x}$ junction. The junction has been fabricated at the University of Twente and has a $\mathrm{Nb} / \mathrm{Al} / \mathrm{AlO}_{x} / \mathrm{Al} / \mathrm{Nb}$ multilayer structure without trapping layers: $300 \mathrm{~nm}$ of $\mathrm{Nb}$ is sputtered on the oxidized $\mathrm{Si}$ substrate, followed by $5 \mathrm{~nm}$ of $\mathrm{Al}$, together comprising the base electrode. The Al is thermally oxidized, forming a barrier with an estimated thickness of $1 \mathrm{~nm}$ and a normal resistivity of $6 \times 10^{-6} \Omega \mathrm{cm}^{2}$. In the same vacuum run the counter electrode is sputtered, consisting of $4 \mathrm{~nm}$ of Al, $200 \mathrm{~nm}$ of $\mathrm{Nb}$, and a protective layer of $5 \mathrm{~nm}$ of Al. After patterning and insulation of the edges, the counter electrode is partially covered by a $500 \mathrm{~nm}$ thick $44 \mu \mathrm{m} \times$ $44 \mu \mathrm{m}$ contact pad of $\mathrm{Nb}$ wiring. The width of the connecting leads is $5 \mu \mathrm{m}$.

The medsurements have been performed at a temperature of $1.1 \mathrm{~K}$ in a parallel magnetic field of $125 \mathrm{G}$. The junction is irradiated by an ${ }^{55} \mathrm{Fe}$ source. For details of the experimental setup, see Ref. [5].

In Fig. 2 the result of a typical measurement is shown. 


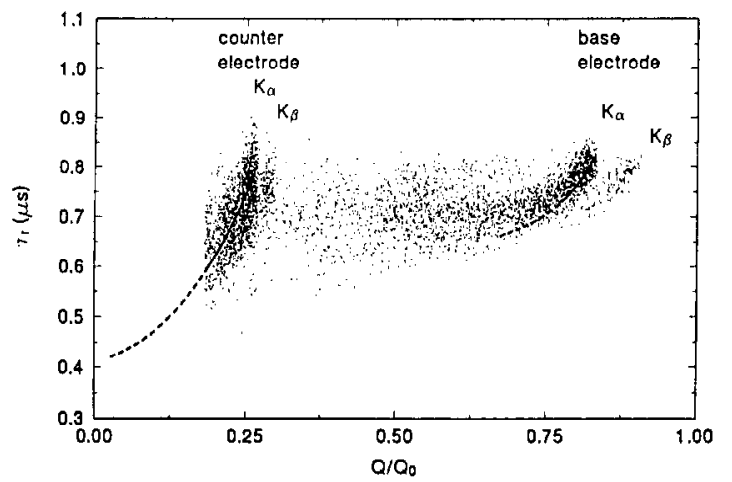

Fig. 2. Risetime-charge diagram showing experimental data (scattered points) and theoretical curves (solid and dashed lines).

The left cloud is attributed to X-ray events in the counter electrode, the right cloud to X-ray events in the base electrode. The doublet structure due to the $\mathrm{Mn}-\mathrm{K}_{\alpha}$ and $\mathrm{Mn}-\mathrm{K}_{\beta}$ pcaks is clcarly visible. $\Lambda$ particularly striking feature is the elongated bent shape of the base electrode event cloud. We carefully checked that the distribution of these events is not correlated with the variation of any experimental parameter. We therefore believe the bent shape is due to intrinsic junction propertics. In the corresponding pulse height spectrum the FWHM of the base electrode $\mathrm{Mn}-\mathrm{K}_{r}$ peak amounts to a rather disappointing value of about $150 \mathrm{eV}$. However, by removing events with a risetime smaller than $0.8 \mu \mathrm{s}$, the FWHM can be reduced to below $100 \mathrm{eV}$. The FWHM due to the electronic noise is approximately $50 \mathrm{eV}$.

We have fitted the model to the experimental data by assuming that the elongated distributions of X-ray events are due to spatial variation of the junction time constants. Variation of a single parameter, or of various independent parameters simultaneously, does not give satisfactory results. It turns out it is particularly difficult to reproduce the full bent shape of the base electrode cloud, while simultaneously fitting the counter electrode cloud. We find that a reasonable fit can only be obtained if both the tunneltime $\tau_{t, 2}$ and the lifetime $\tau_{1,2}$ in the counter electrode are varied over a large range in a correlated manner. In Fig. 2 a fit is shown (solid and dashed lines) with $N_{0}=$ $2.4 \times 10^{6}, \tau_{\mathrm{t}, 1}=1.0 \mu \mathrm{s}$, and $\tau_{1,1}=1.9 \mu \mathrm{s}$. The correlated variation of $\tau_{1,2}$ and $\tau_{\mathrm{t}, 2}$ is given by $0.03 \mu \mathrm{s}<\tau_{1,2}<$ $0.48 \mu \mathrm{s}$, and $\tau_{\mathrm{t}, 2}=1.4 \mu \mathrm{s}+3 \tau_{1,2}$. The theoretical curves are dashed for parameter values for which the counter electrode events are obscured by substrate events $(Q(\infty) /$ $Q_{1}<0.2$ ). These have not been recorded. The widths of the event clouds can be accounted for by roughly $\pm 2 \%$ variation of $\tau_{t, 1}$ and $\pm 5 \%$ variation of $\tau_{t, 2}$. Also $\tau_{1,1}$ may be varied by roughly $\pm 5 \%$ without seriously affecting the fit.
Using the value $2 \times 10^{47} \mathrm{~J}^{-1} \mathrm{~m}^{\cdots 3}$ for the single spin density of states of $\mathrm{Nb}[6]$, the value of $\tau_{t, 1}$ obtained from the fit is in approximate agreement with the commonly used theoretical expression [7]. The variation of $\tau_{\mathrm{t} .2}$ cannot be ascribed to inhomogeneity of the tunnel barrier, because then $\tau_{\text {r.l }}$ should vary by the same amount. Since tunneltimes scale with the thickness of the electrodes, the relative variation of $\tau_{t .2}$ may be attributed to the variation in thickness of the counter electrode between 200 and $700 \mathrm{~nm}$, due to the partial coverage by the contact pad. However, the absolute values of $\tau_{1.2}$ differ from theory by at least a factor of 2 . If one assumes that quasiparticle losses occur mainly at interfaces, e.g. due to reduced gap regions, $\tau_{1.2}$ should also scale with the electrode thickness. Variations in $\tau_{1,2}$ should then be positively correlated with variations in $\tau_{1,2}$, as is found from the fit.

\section{Conclusion}

We have shown that simultaneous measurement of the risetime and the total charge, in combination with the presented model, can be useful for gaining quantitative understanding of the behavior of SIS tunnel junctions. We find strong indications that the X-ray energy resolution in our junction is mainly limited by a correlated variation of the tunneltime and the lifetime in the counter electrode. Probably this is caused by variation of its thickness. We therefore plan to improve the energy resolution of our junctions by making the counter electrode more homogeneous.

\section{Acknowledgements}

This work is financially supported by the "Nederlandse Organisatie voor Wetenschappelijk Onderzoek (NWO)".

\section{References}

[1] For a collection of recent papers, see: T.A. Girard, A. Morales and G. Waysand (Eds.), Superconductivity and Particle Detection, Proc. Toledo, Spain (World Scientific, 1994).

[2] M. Kurakado, Nucl. Instr. and Meth. 196 (1982) 275.

[3] N. Rando et al., Nucl. Instr. and Meth. A 313 (1992) 173.

[4] D.J. Goldie et al., Appl. Phys. Lett. 64 (1994) 3169.

[5] J.B. Legrand, Ph.D. thesis, University of Utrecht (1994).

[6] S.B. Kaplan et al., Phys. Rev. B 14 (1976) 4854.

[7] P.A.J. de Korte et al., Proc. SPIE 1743 (1992). 